\title{
Patient with Large Liver Tumor, Bleeding Ulcer and Unstable Angina Treated with Coronary Stenting Followed by Portal Embolization and Major Liver Surgery with Ticagrelor and Aggrastat Prophylaxis
}

Per Sandstrom*, Thomas Gasslander, Bergthor Björnsson, Rickard Bohnmark, Anna Holm, Thomas Muhr and Sofia Sederholm Lawesson

Department of Upper Gastrointestinal Surgery, Institute for Clinical and Experimental Medicine, Linköping, Östergötland, Sweden

"Corresponding author: Sandstrom P, Department of Upper Gastrointestinal Surgery, Institute for Clinical and Experimental Medicine, Linköping, Östergötland, Sweden, Tel: +46 10103 3556; E-mail: Per.sandstrom@liu.se

Rec date: Jun 07, 2016; Acc date: Aug 02, 2016; Pub date: Aug 06, 2016

Copyright: (c) 2016 Sandstrom P, et al. This is an open-access article distributed under the terms of the Creative Commons Attribution License, which permits unrestricted use, distribution, and reproduction in any medium, provided the original author and source are credited.

\begin{abstract}
Background: There is very little data in the literature on how to handle Dual Antiplatelet Therapy (DAPT) and newly adapted Drug Eluting Stents (DES) in patients in need of liver surgery due to malignant disease.

Case report: A man, 79 years of age, with a bleeding hepatocellular carcinoma in a normal liver, bleeding duodenal ulcer and Acute Coronary Syndrome (ACS). Coronary angiography showed significant stenoses in the left main coronary artery (LM), Left Anterior Descending (LAD), first and second diagonal branches (D1, D2), Circumflex (Cx) and Right Coronary Artery (RCA). The patient was treated with Percutaneous Coronary Intervention (PCI) including six everolimus eluting coronary stents, aspirin and ticagrelor followed by portal embolization two months after stenting and another two months later liver resection, bridging with the Glycoprotein Receptor Inhibitor (GPI) tirofiban. The portal embolization was uneventful but there were bleeding complications after liver surgery, calling for very close monitoring of the antithrombotic treatment under these complex conditions.
\end{abstract}

Discussion: Close monitoring and individualization of therapy was essential to make portal embolization and liver tumor surgery possible in this patient with DES and DAPT.

Keywords: Liver surgery; Hepatocellular carcinoma; Antithrombotic; Therapy

\section{Background}

Acute coronary syndrome (ACS) is caused by obstructive coronary artery disease $(\mathrm{CAD})$ complicated by plaque rapture and subsequent activation of the platelets and the coagulation cascade. The treatment of choice according to guidelines is most often percutaneous coronary intervention (PCI) with DES followed by DAPT continued at least one year after the procedure, in order to avoid stent thrombosis and new cardiac events [1]. In patients with malignant liver tumors this may be a problem as tumor treatment needs to be postponed for several months due to the need for DAPT. The timing of surgical liver intervention and other abdominal operations under these conditions is rarely reported in the literature [2]. Regarding heart surgery it is well known that ticagrelor should be stopped at least five days before heart surgery to reduce the risk of bleeding. This case report presents a patient with a large liver tumor and concurrent ACS with advanced CAD.

\section{Case Report}

A 79-year-old man with a history of insulin and metformin treated diabetes mellitus, myocardial infarction (MI) 30 years ago, a Transient Ischemic Attack (TIA) 20 years ago, essential hypertension and radiation treated prostate cancer without signs of recurrence. The patient had a $14 \mathrm{~cm}$ liver tumor in segment 5-8 primarily suspected to be a Hepatocellular Carcinoma (HCC). The segments 2,3 and 4 were non cirrhotic and the liver function tests were normal. He was referred as an emergency case after diagnostic percutaneous liver biopsy at another hospital, resulting in major bleeding. The patient had been observed for two days and given three units of blood before transfer. On arrival in our unit the bleeding was treated with selective embolization of the feeding artery. After embolization the patient developed chest discomfort, ECG abnormalities with signs of ischemia and elevated troponin (hsTnT 81-67-129 ng/L) and he was therefore transferred to the Coronary Care Unit (CCU). He was treated with aspirin and the ADP-receptor inhibitor clopidogrel. This treatment was complicated with an upper gastro intestinal bleeding that required emergent gastroscopy to stop the bleeding, and to make coronary angiography and subsequent PCI possible. The bleeding ulcer was successfully treated with percutaneous embolization of the gastroduodenal artery two days after the liver embolization.

The following coronary angiography showed significant stenoses in the LM coronary artery, all three epicardial coronary arteries; LAD, Cx and RCA. On a multidisciplinary heart conference it was decided that, despite three-vessel-disease, Coronary Artery Bypass Grafting (CABG) was not an option in this case due to the malignancy. It was decided to proceed with PCI. The patient needed six DES to treat all stenoses, and was then put on aspirin $75 \mathrm{mg}$ once daily and ticagrelor $90 \mathrm{mg}$ twice daily. The recovery after these treatments were uneventful and the patient got back to full daily activities (WHO/ECOG=0), including long daily walks. A follow-up echocardiography was normal and bicycle ergometer test showed a $130 \%$ working capacity in relation to his age group. 
Due to the size of the liver tumor and the small size of the future liver remnant segments 1-4 (467 ml; estimated FLR 29\%), a portal embolization was needed [3]. Two months after stenting the patient was therefore readmitted to the cardiology department and ticagrelor treatment was stopped five days before intervention and low dose tirofiban infusion was started in hospital three days before the intervention. The infusion continued until six hours before embolization and restarted six hours afterwards. The portal branches to segment 5-8 were embolized with coils in a percutaneous ipsilateral approach [4]. There were no bleeding complications and the patient was restarted on ticagrelor two days after embolization. Liver laboratory tests showed stable INR and bilirubin but AST/ALT $>40$. This was probably due to previous arterial tumor embolization and was normalized within a few days. Radiological evaluation six weeks after liver embolization showed an increase in eFLR to 37\% (590 ml) acceptable for liver resection. After a total of four months after coronary stenting the patient was planned for liver resection. Ticagrelor was stopped five days before liver surgery and low dose tirofiban infusion started. The infusion continued until six hours before surgery. The operation was uneventful, and a decision was made to restart the tirofiban and heparin infusion six hours postoperatively. During the night the APTT rose to high levels and both infusions were therefore stopped. The morning after there were signs of bleeding and a CT scan showed extravasation on the liver surface and in the urinary bladder. The feeding liver artery was embolized and the patient was only given aspirin and tinzaparin $4500 \mathrm{E} \times 1$ subcutaneously. The bleeding from the urinary bladder was treated with catheter, fluid perfusion and cystoscopy. Daily evaluations with platelet aggregation tests showed a reduced platelet function and therefore no more antiplatelet therapies was added primarily. When the bleeding situation was under control clopidogrel was reinstated. The patient was discharged 3 weeks after surgery, with DAPT (aspirin and clopidogrel) for another 6 months. The pathology report confirmed the diagnosis and showed a $14 \mathrm{~cm}$ radical resected highly differentiated hepatocellular carcinoma. The patient developed a postoperative infection in the perihepatic hematoma that was treated with percutaneous drain. The first follow up radiology 6 months after surgery shows no signs of recurrence and the patient was back in full activity.

\section{Discussion}

Patients with hepatocellular carcinoma have a median survival of less than 6 months without curative surgery [5]. If a curative resection is possible a 5 year survival of more than $50 \%$ may be achieved [6]. Therefore in the right selected patient curative surgery is well motivated.

Improvement in treatment of cardiac and abdominal diseases with percutaneous modalities makes it possible to treat patients with multiple disorders with successful outcome, but with a very delicate balance between the risk of bleeding and the risk of new ischemic events.

In this case, there were a number of things that made the whole process possible. The patient was determined to go through the full treatment and fully informed about the high risk procedures. The liver tumor, a highly differentiated hepatocellular carcinoma was slow growing making it possible to treat the patient with DES, ticagrelor and aspirin. The initial angiographic treatment of the liver tumor and gastroduodenal bleeding was successful without complications or rebleeding. The portal embolization gave enough growth of the FLR and the liver was not cirrhotic. The bleeding after liver surgery was probably due to the combination of tirofiban and heparin and not closes enough monitoring of the APTT.

The use of new antithrombotic treatments puts a high demand on the monitoring of the patient and a close collaboration between different specialties. After PCI using DES, patients should receive DAPT for at least one year as premature withdrawal is associated with a high risk of stent thrombosis and thus of MI and/or death [7-9]. In DES patients requiring DAPT interruption prior to surgery, a shortacting antiplatelet therapy has been proposed as a bridge to surgery but there is a lack of randomized studies comparing different bridging strategies. In two small observational studies of patients with recently implanted DES undergoing urgent surgery a bridging regime with tirofiban with or without concomitant heparin was found successful [10]. Ohers have found increased risk of bleeding as well as of ischemic events after regimes with GPI bridging [11]. Cangrelor is a reversible P2Y12 receptor inhibitor that got approved from regulatory agencies fairly recently. It is administered intravenously with a rapid onset and the offset is more rapid and the binding to the ADP receptor more specific compared with the GPI alternatives. Overall, these characteristics make it a highly desirable agent for bridging therapy. The results of the BRIDGE trial support cangrelor as a valuable bridging agent in the setting of CABG [12]. Whether these findings are also adaptable to different non-cardiac surgeries is still unproven, and this drug was not available at our hospital when this patient was treated with PCI and concomitant surgery, but may be an option in the nearby future.

\section{References}

1. Fleisher LA, Beckman JA, Brown KA, Calkins H, Chaikof EL, et al. (2007) ACC/AHA 2007 Guidelines on perioperative cardiovascular evaluation and care for noncardiac surgery: Executive summary. J Am College Cardiol 116:1707-1732.

2. Cahoon WD, Oswalt AK, Francis KE, Magee LC, Lowe DK (2016) Cangrelor bridge therapy for gastroduodenal biopsy: A case report. J pharm pract.

3. Ribero D, Chun YS, Vauthey JN (2008) Standardized liver volumetry for portal vein embolization. Semin Intervent Radiol 25: 104-109.

4. Ebata T, Yokoyama Y, Igami T, Sugawara G, Takahashi Y, et al. (2012) Portal vein embolization before extended hepatectomy for biliary cancer: current technique and review of 494 consecutive embolizations. Dig Surg 29: 23-29.

5. Verslype C, Van Cutsem E, Dicato M, Arber N, Berlin JD, et al. (2009) The management of hepatocellular carcinoma. Current expert opinion and recommendations derived from the 10th World Congress on Gastrointestinal Cancer, Barcelona, 2008. Ann Oncol 20: vii1-vii6.

6. Cho YK, Rhim H, Noh S (2011) Radiofrequency ablation versus surgical resection as primary treatment of hepatocellular carcinoma meeting the Milan criteria: a systematic review. J Gastroenterol Hepatol 26: 1354-1360.

7. Iakovou I, Schmidt T, Bonizzoni E, Ge L, Sangiorgi GM, et al. (2005) Incidence, predictors, and outcome of thrombosis after successful implantation of drug-eluting stents. Jama 293: 2126-2130.

8. Spertus JA, Kettelkamp R, Vance C, Decker C, Jones PG, et al. (2006) Prevalence, predictors, and outcomes of premature discontinuation of thienopyridine therapy after drug-eluting stent placement: Results from the PREMIER registry. Circulation 113: 2803-2809.

9. Van Werkum JW, Heestermans AA, Zomer AC, Kelder JC, Suttorp MJ, et al. (2009) Predictors of coronary stent thrombosis: the Dutch Stent Thrombosis Registry. Journal of the American College of Cardiology 53: 1399-1409. 
Citation: Sandstrom P, Gasslander T, Björnsson B, Bohnmark R, Holm A, et al. (2016) Patient with Large Liver Tumor, Bleeding Ulcer and Unstable Angina Treated with Coronary Stenting Followed by Portal Embolization and Major Liver Surgery with Ticagrelor and Aggrastat Prophylaxis. J Clin Case Rep 6: 844. doi:10.4172/2165-7920.1000844

Page 3 of 3

10. Savonitto S, D'Urbano M, Caracciolo M, Barlocco F, Mariani G, et al. (2010) Urgent surgery in patients with a recently implanted coronary drug-eluting stent: A phase II study of 'bridging' antiplatelet therapy with tirofiban during temporary withdrawal of clopidogrel. Br J Anaesth 104: 285-291.

11. Alshawabkeh LI, Prasad A, Lenkovsky F, Makary LF, Kandil ES, et al. (2013) Outcomes of a preoperative "bridging" strategy with glycoprotein
IIb/IIIa inhibitors to prevent perioperative stent thrombosis in patients with drug-eluting stents who undergo surgery necessitating interruption of thienopyridine administration. EuroIntervention 9: 204-211.

12. Angiolillo DJ, Firstenberg MS, Price MJ, Tummala PE, Hutyra M, et al. (2012) Bridging antiplatelet therapy with cangrelor in patients undergoing cardiac surgery: a randomized controlled trial. Jama 307: 265-274. 